\title{
Spinal Cord Tract
}

National Cancer Institute

\section{Source}

National Cancer Institute. Spinal Cord Tract. NCI Thesaurus. Code C12995.

White matter in the spinal cord comprising longitudinally oriented nerve fibers (axons) that transmit impulses in both directions: ascending tracts transmit sensory information to the brain from the body, and descending tracts transmit impulses from the brain to the muscles. 